EPJ Web of Conferences 66, 04008 (2014)

DOI: $10.1051 /$ epjconf/ 20146604008

(C) Owned by the authors, published by EDP Sciences, 2014

\title{
Dense nucleonic matter and the renormalization group
}

\author{
Matthias Drews ${ }^{1,2, a}$, Thomas Hell ${ }^{1,2}$, Bertram Klein ${ }^{1}$, and Wolfram Weise ${ }^{1,2}$ \\ ${ }^{1}$ Physik Department T39, Technische Universität München, 85748 Garching, Germany \\ ${ }^{2} E_{C T}{ }^{\star}$, Villa Tambosi, I-38123 Villazzano (Trento), Italy
}

\begin{abstract}
Fluctuations are included in a chiral nucleon-meson model within the framework of the functional renormalization group. The model, with parameters fitted to reproduce the nuclear liquid-gas phase transition, is used to study the phase diagram of QCD. We find good agreement with results from chiral effective field theory. Moreover, the results show a separation of the chemical freeze-out line and chiral symmetry restoration at large baryon chemical potentials.
\end{abstract}

\section{Introduction}

The quest for the critical end point of a first-order chiral transition in the QCD phase diagram is still unsettled. Lattice calculations at imaginary chemical potential seem to disfavor a first-order transition [1]. Model calculations are so far not conclusive. In the Polyakov-loop-extended quark-meson model, studied using the functional renormalization group, the critical endpoint lies in an unphysical region at very small temperatures and outside the range of applicability of the model [2]. In the Polyakov-Nambu-Jona-Lasinio model, the existence of the critical endpoint depends crucially on its input parameters such as the strengths of the axial anomaly and vector couplings [3].

A related question is whether there exists a connection between chiral symmetry restoration and the chemical freeze-out line as determined in a hadron-resonance-gas model analysis of heavy-ion collision data [4]. For very small baryon chemical potentials the chiral crossover lies close to the chemical freeze-out points, not unexpectedly since chemical equilibration requires multi-particle effects and collective phenomena right at the borderline of hadronization mostly into pions [5]. At larger baryon chemical potentials it is mandatory to take all the well-established constraints from nuclear physics into account. A way to do this is to start with a model based on the relevant nucleonic and mesonic degrees of freedom. In order to study chiral restoration, a chiral nucleon-meson model is chosen [6]. No relationship between chiral restoration and chemical freeze-out points is found in this model, at least not in the mean-field approximation [7].

In the following, we will briefly review the chiral nucleon-meson model and its mean-field treatment. Then we strengthen the conclusions by providing a self-consistent treatment of thermal mesonic and nucleonic fluctuations in the framework of the functional renormalization group (FRG). The phase diagram around the nuclear liquid-gas transition is compared with results of calculations using chiral effective field theory [8].

ae-mail: matthias.drews@ph.tum.de

This is an Open Access article distributed under the terms of the Creative Commons Attribution License 2.0, which permits unrestricted use, distribution, and reproduction in any medium, provided the original work is properly cited. 


\section{The chiral nucleon-meson model}

The dominant degrees of freedom around the nuclear liquid-gas transition are nucleons and pions. The pions are combined with a scalar field, $\sigma$, into a four-component vector, $\phi=(\sigma, \pi)$, that transforms under $\mathrm{SO}(4) \cong \mathrm{SU}(2) \times \mathrm{SU}(2)$, with the invariant $\rho=\frac{1}{2}|\phi|^{2}=\frac{1}{2}\left(\sigma^{2}+\pi \cdot \pi\right)$. Moreover, the nucleon is coupled to an isoscalar vector field, $\omega_{\mu}$, generating a repulsive short-range nucleon-nucleon interaction. The Lagrangian of the chiral nucleon-meson model reads [6]:

$$
\begin{aligned}
\mathcal{L}= & \bar{\psi}\left[i \not \partial+g_{s}\left(\sigma+i \gamma_{5} \pi \cdot \tau\right)-g_{v} \gamma^{\mu} \omega_{\mu}+\gamma^{0} \mu\right] \psi+\partial_{[\mu} \omega_{\nu]} \partial^{[\mu} \omega^{\nu]}+\frac{1}{2} m_{v}^{2} \omega_{\mu} \omega^{\mu}+ \\
& +\frac{1}{2} \partial_{\mu} \sigma \partial^{\mu} \sigma+\frac{1}{2} \partial_{\mu} \boldsymbol{\pi} \cdot \partial^{\mu} \boldsymbol{\pi}+U_{\text {mic }}(\rho, \sigma) .
\end{aligned}
$$

While the microscopic potential, $U_{\text {mic }}$, is unknown a priori, the relevant object of interest is the effective potential $U$ at a given temperature and baryon chemical potential with respect to the potential right at the equilibrium point of nuclear matter, $U(T, \mu)-U\left(T=0, \mu=\mu_{c}\right)$. Here, $\mu_{c}=M_{N}-B=923 \mathrm{MeV}$, i. e., the difference of the nucleon mass and the binding energy per nucleon, coincides with the critical chemical potential at the $T=0$ intercept of the liquid-gas phase transition line. In the mean-field approximation the spatial components of the $\omega_{\mu}$ field vanish in order to preserve rotational invariance. In addition, we assume that there is no pion condensate, so only the mean-field values of the $\sigma$ and the $\omega_{0}$ will contribute. The nucleons can be integrated out and the effective potential takes the form

$$
U_{\mathrm{MF}}=U\left(\sigma, \omega_{0}\right)-4 T \int \frac{d^{3} p}{(2 \pi)^{3}} \log \left[1+\mathrm{e}^{-\left(E_{N}(p)-\mu_{\mathrm{eff}}\right) / T}\right]-4 T \int \frac{d^{3} p}{(2 \pi)^{3}} \log \left[1+\mathrm{e}^{-\left(E_{N}(p)+\mu_{\mathrm{eff}}\right) / T}\right],
$$

with $E_{N}(p)^{2}=p^{2}+\left(g_{s} \sigma\right)^{2}$ and $\mu_{\text {eff }}=\mu-g_{v} \omega_{0}$. The potential $U\left(\sigma, \omega_{0}\right)$ is chosen in such a way as to reproduce nuclear physics constraints [7]. The mean-field potential $U_{\mathrm{MF}}$ is then minimized as a function of $\sigma$ and $\omega_{0}$. Next we explain how to go beyond this mean field approximation.

\section{Adding fluctuations}

In order to incorporate fluctuations in a self-consistent manner, the functional renormalization group (FRG) method is used ([9] and references therein). The effective action, $\Gamma_{k}$, at a renormalization scale $k$, interpolates in this framework between the microscopic action, $S=\Gamma_{k=\Lambda}$, at a cutoff scale $\Lambda$ and the full effective action, $\Gamma_{\text {eff }}=\Gamma_{k=0}$, with all fluctuations integrated out. The flow of this action, as the scale $k$ is lowered, is governed by Wetterich's flow equation [10]:

$$
k \partial_{k} \Gamma_{k}=\bigotimes \oint=\frac{1}{2} \operatorname{Tr} \frac{k \partial_{k} R_{k}}{\Gamma^{(2)}+R_{k}}
$$

The trace is taken over all fields, as well as their momenta and internal degrees of freedom. $\Gamma^{(2)}$ is the second derivative of the effective action with respect to the fields, such that the last expression is the full propagator, with an insertion of a regulator function $R_{k}$. This regulator function ensures that only fluctuations around $k$ are contributing at that scale. The regulator gives low momentum modes an effective mass of order $k^{2}$. In practice, $R_{k}$ is chosen to be the regulator proposed by Litim [11] for application at finite temperatures $[12,13]$ :

$$
R_{k}=\left(k^{2}-\boldsymbol{p}^{2}\right) \cdot \theta\left(k^{2}-\boldsymbol{p}^{2}\right)
$$


The potential is fitted to reproduce nuclear physics constraints such as the liquid-gas transition. The effective potential is therefore expanded around $T=0$ and $\mu=\mu_{c}$ and the flow of

$$
\bar{\Gamma}_{k}=\Gamma_{k}(T, \mu)-\Gamma_{k}\left(0, \mu_{c}\right)
$$

is computed. Thermal fluctuations around the liquid-gas transition are treated self-consistently in this way [12]. In the local-potential approximation and in leading order in the derivative expansion, the flow equation for the effective action, $\bar{\Gamma}_{k}$, reduces to an equation for the effective potential, $\bar{U}_{k}$. The trace can be computed explicitly and the flow equation for the effective potential becomes

$$
\partial_{k} \bar{U}_{k}=f(T, \mu)-f\left(0, \mu_{c}\right),
$$

where

$$
f(T, \mu)=\frac{k^{4}}{12 \pi^{2}}\left\{\frac{3\left[1+2 n_{B}\left(E_{\pi}\right)\right]}{E_{\pi}}+\frac{1+2 n_{B}\left(E_{\sigma}\right)}{E_{\sigma}}-\frac{8\left[1-n_{F}\left(E_{N}, \mu_{\mathrm{eff}}\right)-n_{F}\left(E_{N},-\mu_{\mathrm{eff}}\right)\right]}{E_{N}}\right\} .
$$

Here

$$
\begin{gathered}
E_{\pi}^{2}=k^{2}+m_{\pi}^{2}, \quad E_{\sigma}^{2}=k^{2}+m_{\sigma}^{2}, \quad E_{N}^{2}=k^{2}+2 g_{s}^{2} \rho, \quad m_{\pi}^{2}=U_{k}^{\prime}(\rho), \quad m_{\sigma}^{2}=U_{k}^{\prime}(\rho)+2 \rho U_{k}^{\prime \prime}(\rho), \\
m_{N}=g_{s} \sigma, \quad \mu_{\mathrm{eff}}=\mu-g_{v} \omega_{0, k}, \quad n_{B}(E)=\frac{1}{\mathrm{e}^{\beta E}-1} \quad \text { and } n_{F}(E, \mu)=\frac{1}{\mathrm{e}^{\beta(E-\mu)}+1} .
\end{gathered}
$$

The flow equation for the background vector field $\omega_{0, k}$ leads to the integral equation

$$
\omega_{0, k}=\frac{2 g_{v}}{3 \pi^{2} m_{v}^{2}} \int_{k}^{\Lambda} d \tilde{k} \tilde{k}^{4} \frac{\partial}{\partial \mu} \frac{n_{F}\left(E_{N}(\tilde{k}), \mu_{\mathrm{eff}}\right)+n_{F}\left(E_{N}(\tilde{k}),-\mu_{\mathrm{eff}}\right)}{E_{N}(\tilde{k})} .
$$

The effective potential is fixed in such a way that the nuclear saturation density, the binding energy, the surface tension of a nuclear droplet, and the compression modulus obtained from the full potential agree with empirical data. The flow equation is then solved for temperatures and baryon chemical potentials around the liquid-gas phase transition using a numerical method on a discretized grid [14].

\section{Results and discussion}

The nuclear liquid-gas phase transition has been studied extensively within the framework of chiral effective field theory ( $\chi$ EFT, [15] and references therein). In the left plot of Fig. 1 the first-order line and the critical point are shown, as computed in the nucleon-meson model, both at mean-field level and including fluctuations. They are compared with a $\chi$ EFT computation [8] which takes into account all one- and two-pion exchange processes, as well as three-body forces and $\Delta$-isobar excitations. With fluctuations, the transition is bent away from the mean-field curve to higher chemical potentials, in very good agreement with $\chi$ EFT results. This is remarkable, given the very different approaches. Whereas the temperature of the critical point is $15.1 \mathrm{MeV}$ in $\chi \mathrm{EFT}$, it is shifted to a value of $T_{c}=$ 18.3 MeV in the RG treatment of the nucleon-meson model, in good agreement with empirical results [16].

In order to address the entanglement between chemical freeze-out points and chiral restoration, the chiral condensate is studied as a function of temperature and chemical potential. In the nucleon-meson model, the chiral condensate is proportional to the expectation value of $\sigma$. In the plot on the righthand side of Fig. 1, contour lines of $\sigma$ normalized to its vacuum expectation value, $f_{\pi}$, are shown. In the whole area of temperatures up to $100 \mathrm{MeV}$ and baryon chemical potentials smaller than about 

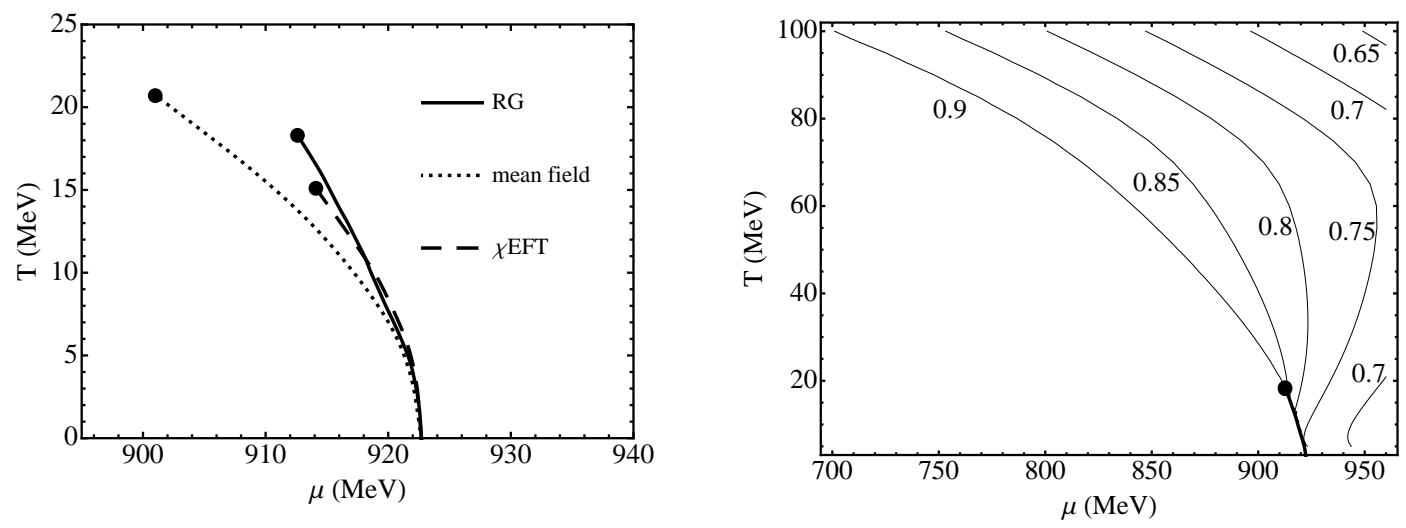

Figure 1. Left: liquid-gas phase transition in $\chi$ EFT [8] (dashed) and in the chiral nucleon-meson model both at mean-field level (dotted) and with fluctuations (solid). Right: contour plots of $\sigma / f_{\pi}$ representing the chiral order parameter. Within the region of applicability, the condensate is non-zero throughout and chiral symmetry is spontaneously broken.

$1 \mathrm{GeV}$, the chiral order parameter $\sigma / f_{\pi}$ still exceeds 0.65 and chiral symmetry is not restored in its trivial Wigner-Weyl realization. The line at which chiral symmetry is restored must therefore intersect the $\mu$-axis at considerably larger baryon chemical potentials and is therefore well separated from the nuclear liquid-gas phase transition, as it should be. In the region of applicability, there is no sign of a chiral first-order phase transition. This demonstrates the importance of taking the constraints from nuclear physics properly into account in calculations modeling the QCD phase diagram.

\section{References}

[1] P. de Forcrand, O. Philipsen, JHEP 0701:077 (2007)

[2] T. Herbst, J. Pawlowski, B.-J. Schaefer, Phys. Lett. B 696, 58 (2011)

[3] K. Fukushima, Phys. Rev. D 77, 114028 (2008),

N. Bratovic, T. Hatsuda, W. Weise, Phys. Lett. B 719, 131 (2013),

T. Hell, K. Kashiwa, W. Weise, J. Mod. Phys. 4, No. 5, 644 (2013)

[4] A. Andronic, P. Braun-Munzinger, J. Stachel, Phys. Lett. B 673, 2, 142 (2009)

[5] P. Braun-Munzinger, J. Stachel, C. Wetterich, Phys. Lett. B 596, 61 (2004).

[6] J. Berges, D.-U. Jungnickel, C. Wetterich, Int. J. Mod. Phys. A 18, 3189 (2003)

[7] S. Floerchinger, C. Wetterich, Nucl. Phys. A 890-891, 11 (2012)

[8] S. Fiorilla, N. Kaiser, W. Weise, Nucl. Phys. A 880, 65 (2012)

[9] P. Kopietz, L. Bartosch, F. Schütz, Lect. Notes Phys. 798 (2010) (Springer, Berlin \& Heidelberg)

[10] C. Wetterich, Phys. Lett. B 301, 90 (1993)

[11] D. Litim, Phys. Rev. D 64, 105007 (2001)

[12] D. Litim, J. Pawlowski, JHEP 0611:026 (2006)

[13] J.-P. Blaizot et al., Nucl. Phys. A 784, 376 (2007)

[14] J. Adams, et al., Mod. Phys. Lett. A 10, 2367 (1995)

[15] J. Holt, N. Kaiser, W. Weise, Prog. Part. Nucl. Phys, in print (2013), arXiv: 1304.6350

[16] V. A. Karnaukhov et al., Phys. Atom. Nucl. 712067 (2008) 